\title{
Dark Matter Hunter
}

\author{
XinRan Liu develops technology for dark matter detectors, which he \\ hopes will soon be spotting these mysterious particles.
}

\author{
By Katherine Wright
}

X inRan Liu calls his career in dark matter physics a "happy accident." As he was about to apply for graduate school studies in nuclear fusion-a topic he was excited about exploring for its potential to create green energy-Liu noticed that his CV contained a long list of particle-physics-related skills and experience rather than nuclear physics ones. At the last minute, he decided to switch fields. Instead of pursuing nuclear fusion, he chose a path in experimental particle physics, working as a Ph.D. student on the neutrinoless double-beta decay detector for SuperNEMO, an experiment that is searching for possible evidence that neutrinos are their own antiparticles (see Viewpoint: The Hunt for No Neutrinos).

Liu, who works at the University of Edinburgh, UK, is now affiliated with another particle detector-LUX-ZEPLIN at the Sanford Underground Research Facility in South Dakota. This experiment is searching for dark matter particles-the invisible stuff that holds the Universe's galaxies together. Scientists have observed dark matter's gravitational effect on other objects but have yet to see it directly in a lab. Physics spoke to Liu about his passion for dark matter, when he thinks dark matter might be

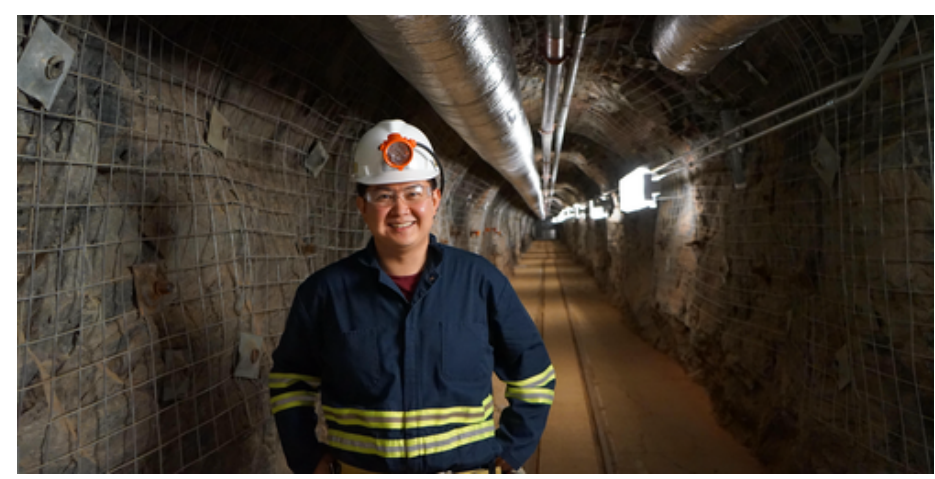

Credit: X. Liu/University of Edinburgh detected, and what will happen if these elusive particles aren't found with current technologies.

All interviews are edited for brevity and clarity.

\section{How have scientists tried to spot dark matter?}

There are three main ways to search for dark matter: make it, shake it, or break it. Make-it experiments smash particles together in a high-energy particle collider and look for dark matter signatures in the debris. Break-it experiments search for dark matter annihilating with its antimatter particle. And shake-it experiments aim to detect the impacts of dark matter on a detector. The shake-it method will likely provide the most definitive detection of dark matter, as it makes a direct measurement of dark matter's interaction with a detector; the other methods look for the products of collisions involving dark matter, an indirect probe. For shake-it methods, the most powerful to date involves a xenon time projection chamber-a detector that uses electric and magnetic fields to reconstruct particle trajectories through liquid xenon.

\section{Why a xenon time projection chamber?}

Xenon is easy to purify in large quantities. It's also transparent to its own scintillation light-the light it emits when it's hit with an incoming particle-so we can detect signals produced anywhere in a xenon chamber.

\section{When do you think a dark matter detection might happen?}

Searching for dark matter is like trying to find a unicorn in a forest. Different theories have different ideas for where the unicorn might be hiding-it might be hiding behind a waterfall or behind a rocky outcrop. The detection experiments work by clearing areas of this forest, crossing out places on the list where the unicorn wasn't found. 
At the end of last year, the scientists behind the XENON1T experiment reported a tantalizing signal that might be dark matter. But the signal had a statistical significance of 3.5 sigma, which, for particle physics, isn't high enough to claim a discovery. To convince everyone that this signal is real, we will need more data. Luckily, next year the XENON experiment and the experiment that I work on, LUX-ZEPLIN, will start taking data again but this time with significantly larger detectors. With the increase in detector size, we should have a good chance of determining if the signal XENON saw is real or a statistical fluke.

\section{How much bigger will the upgraded detectors be?}

The LUX-ZEPLIN detector will contain 10 tons of liquid xenon, 10 times the amount as in the previous XENON experiment, known as XENON1T. We're also placing the xenon in vessels made with the world's cleanest titanium, which helps cut down the noise from radioactivity.

\section{What if these upgraded detectors don't unequivocally find dark matter?}

If that happens, we plan to build one even bigger detector. That detector will have 50 to 100 tons of liquid xenon. That detector, though, will likely be the last of these large-scale xenon detectors.

The reason to stop at a 100-ton detector is that a detector of that size will be sensitive to the neutrino floor, an irreducible background that will swamp the measurements. Reaching the neutrino floor doesn't mean that we will have to give up searching for dark matter, but new techniques will be needed after that point.

\section{And what would it mean for you, and for physics, if dark matter does show up when you turn the detectors on next year?}

It would be amazing; I would definitely be popping open some champagne bottles. And I am sure that some physicists would be heading straight to Sweden to receive a Nobel Prize.

More seriously, I think that such a discovery would open up a new chapter in particle physics, potentially taking us beyond our current theoretical models. Actually, scrap the chapter-one chapter might not be enough. If we find dark matter, we will likely have to write a whole new book of physics, since $85 \%$ of the Universe's mass is dark matter. But chapter or book, a discovery will make it a very exciting time to be a physicist, that's for sure.

Katherine Wright is the Deputy Editor of Physics. 